\title{
Statin Utilization among Patients with Acute Coronary Syndrome: Systematic Review
}

\author{
Pramitha Esha Nirmala Dewi ${ }^{1,2 \star}$, Montarat Thavorncharoensap ${ }^{2}$ \\ ${ }^{1}$ Department of Pharmacy Profession, Faculty of Medicine and Health Sciences, Universitas Muhammadiyah Yogyakarta, \\ Bantul, Indonesia; '2Social, Economic and Administrative Pharmacy Graduate Program, Faculty of Pharmacy, Mahidol \\ University, Bangkok, Thailand
}

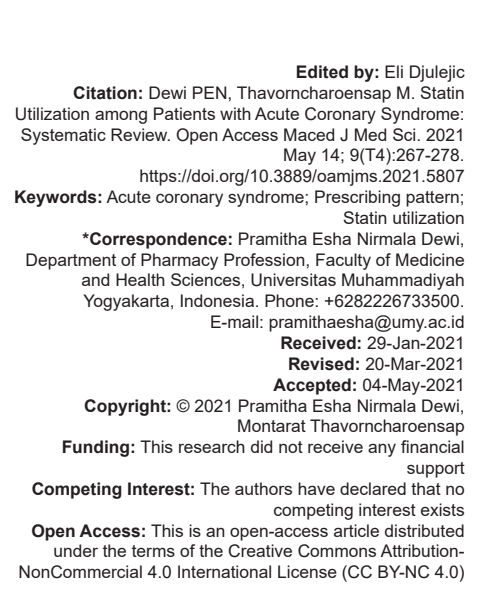

\section{Abstract}

BACKGROUND: The early use of statin with intensive regimen has been recommended by the recent guidelines as the prevention of acute coronary syndrome (ACS) related events among the high-risk patients. Meanwhile, the inconsistent statin utilization for targeted patient in current practice is still an issue.

AIM: This study aims to review the utilization rate of statin among patients with ACS.

METHODS: A systematic search of relevant studies published between inceptions to June 2020 was conducted in PubMed. Patients and intervention domains were used to build up the searching formula. A study was eligible for inclusion if it was an original study of patients with ACS and it examined the utilization of statin. The risk of bias was assessed using Axis and NOS checklist.

RESULTS: Among the 49 eligible studies, 38 were cohort studies while the others were cross-sectional studies. The utilization rate of statin at hospital admission ranged from $16 \%$ to $61 \%$ while $25 \%$ to $75 \%$ during the hospitalization Of the total studies, 35 studies reported the statin rate at discharge ranging from $58 \%$ to $99 \%$. Almost all studies revealed the reduction of statin utilization rate along the follow-up period. The number of statins prescribed was found to be lower among female and elderly patients.

CONCLUSION: Despite the established benefits of statin among patients with ACS, our study revealed that statin was underutilized for secondary prevention after ACS. To improve patients' clinical outcomes with ACS, efforts should be made to increase optimal treatment and compliance with a statin.

\section{Introduction}

The number of death and disability-adjusted life year loss due to the cardiovascular related disease has been widely reported worldwide [1]. The current guidelines recommended the use of statin as the major therapy for atherosclerotic cardiovascular disease (ASCVD) as well as the acute coronary syndrome (ACS) [2]. The primary and secondary prevention purpose of statin prescribing has been applied for patients with ACS [3]. The effect of low-density lipoprotein cholesterol (LDL-c) level reduction is closely related to the diminishing risk of cardiovascular events recurrences among ACS patients [4], [5], [6]. The guideline from American Heart Journal had given their recommendation to initiate or continue statin therapy among patients with clinical or high-risk symptoms of ASCVD since 2013 [4] and still stated in the updated version [2], [5]. Current evidence also revealed that statin could prevent major adverse cardiac events, cardiac death, and re-hospitalization among ACS patients [6], [7], [8], [9], [10]. Although the guidelines and current evidence consistently revealed the benefits of statin among the ACS patients [2], [4], [5], [10], the actual rate of statin utilization was also an issue of concerns. To date, several studies were conducted to examine the rate of statin utilization among the ACS patients in current practice. Therefore, we performed a systematic review to describe statin utilization rate among patients with ACS.

\section{Methods}

\section{Search strategy and eligibility criteria}

Relevant studies were identified from the PubMed database (from inception to June 2020). Patients (P) and Intervention (I) domains were used to build up the searching formula as follows: P- "Acute Coronary Syndrome" [Mesh]; I- "HydroxymethylglutaryCoA Reductase Inhibitors" [Mesh], statin, atorvastatin, simvastatin, rosuvastatin, pitavastatin, pravastatin, and lovastatin. The two domains were combined with AND. Study selection was performed independently 
by two reviewers. A study was eligible for inclusion if; (1) it was an original study conducted among patients with ACS, and (2) it examined the utilization of statin. A study was subsequently excluded if; (1) it was published in non-English language; (2) qualitative study; (3) interventional study; and (4) inaccessible of the full text.

\section{Data extraction and quality assessment}

The predesigned data extraction form was used by the reviewers to extract the data independently. Negotiation and consensus were done among the reviewers to resolve any disagreement. For each included full paper, the authors extracted the following data; bibliography details; setting; study design; characteristics of patients; statin utilization at hospital admission, during hospitalization, discharge and after hospital discharge; the pattern of statin utilization; and factors affecting statin utilization.

The quality assessment of all selected studies was conducted using the standard checklist to set up a good standard for the selected articles, such as the Axis checklist (for cross-sectional study) [11] and the Newcastle-Ottawa (NOS) checklist (for cohort study) [12]. The Axis checklist consisted of 20 questions, classified into the quality of introduction (Q1), study design (Q2), sample size justification (Q3), target population (Q4), sampling frame (Q5), sample selection (Q6), addressing the non-responders (Q7), measurement validity (Q8), measurement reliability (Q9), statistics (Q10), overall methods (Q11), raw data (Q12), response rate (Q13, Q14), the internally consistent result (Q15), comprehensive description of results (Q16), justified discussions and conclusions (Q17), limitations (Q18), conflicts of interest (Q19), and ethical approval (Q20) [11]. The NOS checklist covered quality assessment related to the selection process (4 questions), comparability in the analysis process (1 question), and outcome reported (3 questions) [12].

In terms of the NOS scale, the number of stars represented the quality of cohort studies with 8-9 stars representing good quality, 6-7 stars representing moderate quality, and less than 6 stars representing low-quality [12].

\section{Data analysis}

Characteristics of each included study were described. The utilization of statin was tabulated to identify patterns across the included studies. Utilization at each time point (i.e., before hospitalization, in-hospital, discharge, and follow-up period) was also reported and summarized as a trend of statin use over time.

\section{Results}

\section{Study selection}

A total of 252 studies were identified from the PubMed database. Among those studies, 100 studies were excluded after screening titles and abstracts. Thirty-seven studies were further excluded due to inaccessible of full-text. After screening full-text studies, 66 studies were excluded from the study (not examining the statin utilization-42, review articles-17, interventional studies-4, and not reporting statin utilization among ACS patients-3). Finally, 49 studies were included in this systematic review [Figure 1].

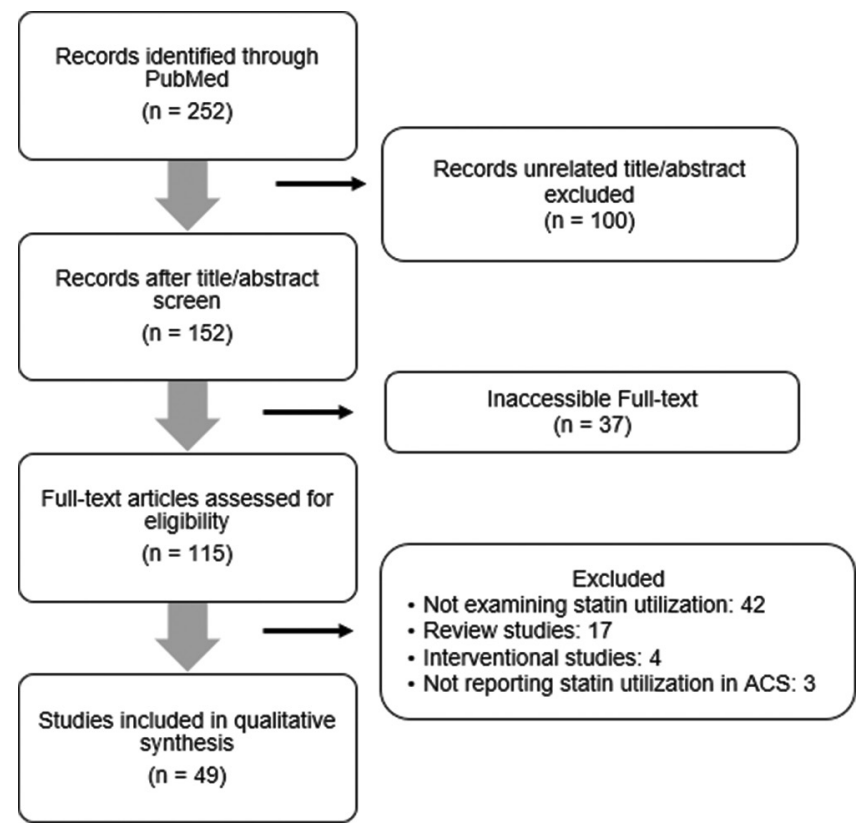

Figure 1: Flow chart for study selection

\section{Study quality}

Among the 41 cross-sectional studies assessed by the Axis checklist, all those studies had "Yes" answer for questions number 1, 2, 4, 5, 6, 9, 10, $11,12,15,16$, and 17 and "No" answer for question number 3 and 14. Eighteen studies did not measure and categorize the non-responders [6], [7], [9], [13], [14], [15], [16], [17], [18], [19], [20], [21], [22], [23], [24], [25], [26]. There were nine studies [10], [18], [24], [27], [28], [29], [30], [31], [32] collecting data on statin use directly from the patients either by interview or selfreporting. By assessing the quality among the selected studies related to question number 13 , missing data/ loss to follow-up was higher than $20 \%$ in the three studies [28], [33], [34]. Referring to question number 18, six studies [8], [15], [21], [35], [36], [37] did not report their study limitation in the discussion part. Thirteen out of 41 studies declared their conflict of interest according to question number 19 in the checklist [9], 
[10], [16], [18], [19], [24], [26], [28], [33], [34], [38], [39], [40]. Twelve studies did not receive the ethic committee approval nor the participant consent [6], [8], [14], [19], [21], [24], [25], [26], [33], [37], [38], [41]. The details of the assessment are presented in Table 1. The eight cohort studies assessed by the NOS checklist, three studies had eight stars [42], [43], [44] and the remaining four studies had nine stars [45], [46], [47], [48], [49], representing high quality. The details of the assessment are shown in Table 2.

\section{Statin prescribing pattern}

This systematic review described the pattern of statin utilization in the ten studies [9], [16], [20], [27], [30], [33], [35], [37], [41], [50], which was prescribed with another ACS medication such as antiplatelet, angiotensin-converting enzyme inhibitor/angiotensin receptor blocker (ACEI/ARB), and beta-blocker as summarized by Table 3. Seven studies [9], [20], [33], [35], [37], [50] reported the use of statin together with aspirin, beta-blockers, and ACEI/ARB, which was considered as the evidence-based treatment of secondary prevention among the ACS patients. The utility rate of such evidence-based treatment varied from $25 \%$ [37] to $86.7 \%$ [50]. The use of statin along with beta-blocker and antiplatelet was reported in five studies [20], [30], [35], [37], [50], with the ranges between $10.1 \%$ [20] and $93.2 \%$ [30] at discharge. The combination therapy between statin and antiplatelet at discharge was examined in the four studies [20], [27], [35], [50] with prescribing rate ranging from $2.6 \%$ [20] to $97.6 \%[50]$.

\section{Study characteristics}

Characteristics of all 49 included studies are shown in Table 4 . The 49 included studies were published from 2008 to 2020. Among the included studies, 13 studies were from Asia, 16 studies from Europe, nine studies from Australia-New Zealand, six from America, one from Africa, and four studies conducted in the selected countries from multiple continents. Of all included studies, nine studies were conducted in multiple countries. Data sources were registry, teaching hospital, specific care unit, national data linkage, and secondary and tertiary hospital. The range of sample sizes varied from 151 to 159,713 . In terms of study design, 38 were cohort, while 11 were cross-sectional studies. All studies except one study [9] examined statin utilization as secondary prevention.

\section{Statin utilization}

Table 5 displays statin utilization along with factors associated with statin utilization. Among all included studies, 14 studies reported the use of statin at hospital admission. Statin utilization at admission

Table 1: Quality assessment of cross-sectional studies

\begin{tabular}{|c|c|c|c|c|c|c|c|c|c|c|c|c|c|c|c|c|c|c|c|c|c|}
\hline Study & Year & Q1 & Q2 & Q3 & Q4 & Q5 & Q6 & Q7 & Q8 & Q9 & Q10 & Q11 & Q12 & Q13 & Q14 & Q15 & Q16 & Q17 & Q18 & Q19 & Q20 \\
\hline Amar et al. [35] & 2008 & Yes & Yes & No & Yes & Yes & Yes & Yes & Yes & Yes & Yes & Yes & Yes & DK & No & Yes & Yes & Yes & No & No & Yes \\
\hline Lee et al. [41] & 2008 & Yes & Yes & No & Yes & Yes & Yes & Yes & Yes & Yes & Yes & Yes & Yes & DK & No & Yes & Yes & Yes & Yes & No & No \\
\hline Vermeer and Bajorek [13] & 2008 & Yes & Yes & No & Yes & Yes & Yes & No & Yes & Yes & Yes & Yes & Yes & DK & No & Yes & Yes & Yes & Yes & No & Yes \\
\hline Bi et al. [27] & 2009 & Yes & Yes & No & Yes & Yes & Yes & Yes & No & Yes & Yes & Yes & Yes & No & No & Yes & Yes & Yes & Yes & No & Yes \\
\hline Wong et al. [37] & 2009 & Yes & Yes & No & Yes & Yes & Yes & Yes & Yes & Yes & Yes & Yes & Yes & No & No & Yes & Yes & Yes & No & No & No \\
\hline Wong et al. [8] & 2009 & Yes & Yes & No & Yes & Yes & Yes & Yes & Yes & Yes & Yes & Yes & Yes & No & No & Yes & Yes & Yes & No & No & No \\
\hline Abdallah et al. [14] & 2010 & Yes & Yes & No & Yes & Yes & Yes & No & Yes & Yes & Yes & Yes & Yes & DK & No & Yes & Yes & Yes & Yes & No & No \\
\hline Melloni et al. [28] & 2010 & Yes & Yes & No & Yes & Yes & Yes & Yes & No & Yes & Yes & Yes & Yes & Yes & No & Yes & Yes & Yes & Yes & Yes & Yes \\
\hline Ramanath et al. [17] & 2010 & Yes & Yes & No & Yes & Yes & Yes & No & Yes & Yes & Yes & Yes & Yes & DK & No & Yes & Yes & Yes & Yes & No & Yes \\
\hline Aliprandi-Costa et al. [18] & 2011 & Yes & Yes & No & Yes & Yes & Yes & No & No & Yes & Yes & Yes & Yes & DK & No & Yes & Yes & Yes & Yes & Yes & Yes \\
\hline Bourdès et al. [50] & 2011 & Yes & Yes & No & Yes & Yes & Yes & Yes & Yes & Yes & Yes & Yes & Yes & No & No & Yes & Yes & Yes & Yes & No & Yes \\
\hline Javed et al. [33] & 2011 & Yes & Yes & No & Yes & Yes & Yes & Yes & Yes & Yes & Yes & Yes & Yes & Yes & No & Yes & Yes & Yes & Yes & Yes & No \\
\hline The Access Investigators [29] & 2011 & Yes & Yes & No & Yes & Yes & Yes & Yes & No & Yes & Yes & Yes & Yes & No & No & Yes & Yes & Yes & Yes & No & Yes \\
\hline Andrikopoulos et al. [30] & 2012 & Yes & Yes & No & Yes & Yes & Yes & Yes & No & Yes & Yes & Yes & Yes & No & No & Yes & Yes & Yes & Yes & No & Yes \\
\hline Ranasinghe et al. [19] & 2012 & Yes & Yes & No & Yes & Yes & Yes & No & Yes & Yes & Yes & Yes & Yes & DK & No & Yes & Yes & Yes & Yes & Yes & No \\
\hline Wai et al. [36] & 2012 & Yes & Yes & No & Yes & Yes & Yes & Yes & Yes & Yes & Yes & Yes & Yes & DK & No & Yes & Yes & Yes & No & No & Yes \\
\hline Yusuf et al. [7] & 2012 & Yes & Yes & No & Yes & Yes & Yes & No & Yes & Yes & Yes & Yes & Yes & DK & No & Yes & Yes & Yes & Yes & No & Yes \\
\hline Andrikopoulos et al. [30] & 2013 & Yes & Yes & No & Yes & Yes & Yes & Yes & Yes & Yes & Yes & Yes & Yes & No & No & Yes & Yes & Yes & Yes & No & Yes \\
\hline Huffman et al. [56] & 2013 & Yes & Yes & No & Yes & Yes & Yes & Yes & Yes & Yes & Yes & Yes & Yes & No & No & Yes & Yes & Yes & Yes & No & Yes \\
\hline Kassab et al. [15] & 2013 & Yes & Yes & No & Yes & Yes & Yes & No & Yes & Yes & Yes & Yes & Yes & DK & No & Yes & Yes & Yes & No & No & Yes \\
\hline Shehab et al. [6] & 2013 & Yes & Yes & No & Yes & Yes & Yes & No & Yes & Yes & Yes & Yes & Yes & DK & No & Yes & Yes & Yes & Yes & No & No \\
\hline Gausia et al. [20] & 2014 & Yes & Yes & No & Yes & Yes & Yes & No & Yes & Yes & Yes & Yes & Yes & DK & No & Yes & Yes & Yes & Yes & No & Yes \\
\hline Grey et al. [71] & 2014 & Yes & Yes & No & Yes & Yes & Yes & Yes & Yes & Yes & Yes & Yes & Yes & No & No & Yes & Yes & Yes & Yes & No & Yes \\
\hline Jin et al. [31] & 2014 & Yes & Yes & No & Yes & Yes & Yes & Yes & No & Yes & Yes & Yes & Yes & No & No & Yes & Yes & Yes & Yes & No & Yes \\
\hline Maggioni et al. [38] & 2014 & Yes & Yes & No & Yes & Yes & Yes & Yes & Yes & Yes & Yes & Yes & Yes & DK & No & Yes & Yes & Yes & Yes & Yes & No \\
\hline Pereira et al. [54] & 2014 & Yes & Yes & No & Yes & Yes & Yes & Yes & Yes & Yes & Yes & Yes & Yes & No & No & Yes & Yes & Yes & Yes & No & Yes \\
\hline Shimony et al. [16] & 2014 & Yes & Yes & No & Yes & Yes & Yes & No & Yes & Yes & Yes & Yes & Yes & DK & No & Yes & Yes & Yes & Yes & Yes & Yes \\
\hline Wang et al. [51] & 2014 & Yes & Yes & No & Yes & Yes & Yes & Yes & Yes & Yes & Yes & Yes & Yes & No & No & Yes & Yes & Yes & Yes & No & Yes \\
\hline Anzai et al. [23] & 2015 & Yes & Yes & No & Yes & Yes & Yes & No & Yes & Yes & Yes & Yes & Yes & DK & No & Yes & Yes & Yes & Yes & No & Yes \\
\hline Ghadri et al. [21] & 2015 & Yes & Yes & No & Yes & Yes & Yes & No & Yes & Yes & Yes & Yes & Yes & DK & No & Yes & Yes & Yes & No & No & No \\
\hline Kassaian et al. [32] & 2015 & Yes & Yes & No & Yes & Yes & Yes & No & No & Yes & Yes & Yes & Yes & DK & No & Yes & Yes & Yes & Yes & No & Yes \\
\hline et al. [22] & 2015 & Yes & Yes & No & Yes & Yes & Yes & No & Yes & Yes & Yes & Yes & Yes & DK & No & Yes & Yes & Yes & Yes & No & Yes \\
\hline Selby et al. [9] & 2015 & Yes & Yes & No & Yes & Yes & Yes & No & Yes & Yes & Yes & Yes & Yes & No & No & Yes & Yes & Yes & Yes & Yes & Yes \\
\hline Gunnell et al. [39] & 2016 & Yes & Yes & No & Yes & Yes & Yes & Yes & Yes & Yes & Yes & Yes & Yes & No & No & Yes & Yes & Yes & Yes & Yes & Yes \\
\hline Boccara et al. [24] & 2017 & Yes & Yes & No & Yes & Yes & Yes & No & No & Yes & Yes & Yes & Yes & DK & No & Yes & Yes & Yes & Yes & Yes & No \\
\hline Eisen et al. & 2017 & Yes & Yes & No & Yes & Yes & Yes & Yes & No & Yes & Yes & Yes & Yes & No & No & Yes & Yes & Yes & Yes & Yes & Yes \\
\hline Khedri et al. [10] & 2017 & Yes & Yes & No & Yes & Yes & Yes & Yes & Yes & Yes & Yes & Yes & Yes & No & No & Yes & Yes & Yes & Yes & Yes & Yes \\
\hline Boklage et al. [26] & 2018 & Yes & Yes & No & Yes & Yes & Yes & No & Yes & Yes & Yes & Yes & Yes & DK & No & Yes & Yes & Yes & Yes & Yes & No \\
\hline Hoedemaker et al. [25] & 2018 & Yes & Yes & No & Yes & Yes & Yes & No & Yes & Yes & Yes & Yes & Yes & DK & No & Yes & Yes & Yes & Yes & No & No \\
\hline Hao et al. [34] & 2019 & Yes & Yes & No & Yes & Yes & Yes & Yes & Yes & Yes & Yes & Yes & Yes & Yes & No & Yes & Yes & Yes & Yes & Yes & Yes \\
\hline Desta et al. [52] & 2020 & Yes & Yes & No & Yes & Yes & Yes & Yes & Yes & Yes & Yes & Yes & Yes & DK & No & Yes & Yes & Yes & Yes & No & Yes \\
\hline
\end{tabular}


ranged from $11.43 \%$ to $94.8 \%$. Unfortunately, there were only two studies whose statin utilization at admission was more than 50\% [6], [47]. In terms of statin utilization during hospitalization, many studies (7 out of 11 studies) reported that statin utilization was higher than 80\% [22], [29], [30], [32], [34], [51], [52]. We found that most of the selected studies (39 studies) measured statin utilization rates at hospital discharge. Statin utilization at discharge varied from $20 \%$ to $99 \%$. It should be noted that 29 studies (74\%) reported that more than $80 \%$ of ACS patients received statin at hospital discharge.

Among the included studies, 18 studies reported statin use in a specified follow-up period after discharge. Most of those studies (17 of 18) reported statin use at 6 and/or 12 months as the follow-up time points. During the follow-up period, statin utilization rate ranged between $24.7 \%$ [31] and $94 \%$ [49]. The lowest rate $(24.7 \%)$ of statin utilization during the follow-up period was reported among elderly patients $(\geq 65$ years old) [31].

\section{Table 2: Quality assessment of cohort studies}

\begin{tabular}{|c|c|c|c|c|c|c|c|c|c|c|}
\hline \multirow[t]{2}{*}{ Study } & \multirow[t]{2}{*}{ Year } & \multicolumn{3}{|c|}{ Selection } & \multicolumn{2}{|c|}{ Comparability } & \multicolumn{3}{|c|}{ Outcome } & \multirow[t]{2}{*}{ Score } \\
\hline & & & & 4 & $\bar{A}$ & B & & $\begin{array}{l}2 \\
\text { (6 months) }\end{array}$ & 3 & \\
\hline Kim et al. [42] & 2012 & * & & * & * & & * & & & 8 \\
\hline Zeymer et al. [43] & 2013 & * & , & * & * & & * & * & * & 8 \\
\hline Gencer et al. [49] & 2015 & * & & & * & * & * & * & * & 9 \\
\hline Ferreira-González et al. [44] & 2016 & * & & * & * & & * & * & * & 8 \\
\hline Mantel et al. [45] & 2017 & * & & * & * & * & * & * & * & 9 \\
\hline Turner et al. [46] & 2017 & * & & * & * & * & * & * & * & 9 \\
\hline Al-Zakwani et al. [47] & 2018 & * & & & * & * & * & * & * & 9 \\
\hline Sun et al. [48] & 2018 & * & & & * & * & * & * & * & 9 \\
\hline
\end{tabular}

The decreasing trends of statin utilization from discharge time point to follow-up periods were reported in the 11 studies [23], [27], [28], [31], [35], [36], [38], [43], [49], [51], [53]. Only one study conducted by Hoedemaker et al. [25] found that statin utilization slightly increased (85.2-88.1\%) during 30 days of posthospitalization discharge then decreased during 12 months of follow-up period (88.1-84.1\%). Among the 11 studies that reported the decreasing tendency of statin utilization, the average of alteration did not exceed $25 \%$ except for one study conducted by Jin et al. [31].

Several studies reported the utilization rate of statin by age and gender. Of the included studies, seven studies compared statin utilization between male and female groups [6], [13], [21], [34], [39], [41], [54]. All of these studies reported that the use of statin was lower in females than males. At discharge time point, the male group was more likely to receive statin therapy compared to the female $(p<0.001)$ as reported by Lee et al. [41] and Vermeer and Bajorek [13] (OR 3.36; 95\% Cl 1.1110.15). Ghadri et al. [21] reported that the female group was less likely to be prescribed with statins at hospital discharge (85.2\% vs. 89.4\%). Shehab et al. [6] reported that the proportion of male versus females receiving statin at admission was $95.1 \%$ versus $93.6 \%$, and, at discharge, it was $92.1 \%$ versus $88.2 \%$. Females were less likely to receive statin during the hospitalization (94.3\% vs. $95.4 \%)$ and at discharge (90.7\% vs. $93.2 \%)$ compared to male as reported by Hao et al. [34]. Among the STEMI patients, $92.4 \%$ of female participants received statin compared to $93.6 \%$ of males. Similarly, in the NSTE-ACS subgroup, $87.1 \%$ of females and $92.2 \%$ of males received statin therapy [54].

Four studies reported the use of statin utilization by age [23], [31], [41], [54]. All of these studies reported a significantly lower rate of statin utilization among the elderly. Elderly patients aged $\geq 80$ years with NSTE-ACS were much less likely to receive statins (OR $0.35,95 \%$ $\mathrm{Cl}$ 0.19-0.64) at a discharge time point, as reported by Pereira et al. [54]. More specifically, into the age group, Lee et al. [41] reported that patients with ages $<45$, $65-79$, and $\geq 80$ years old were significantly less likely to receive statin compared to patients in the 45-64 age group $(p<0.05)$.

Table 3: Statin prescribing pattern with others acute coronary syndrome medication

\begin{tabular}{|c|c|c|c|c|c|c|c|c|}
\hline Study & Year & $\mathrm{AP}+\mathrm{S}$ & ACEI+S & $\mathrm{BB}+\mathrm{S}$ & $\mathrm{BB}+\mathrm{AP}+\mathrm{S}$ & $\mathrm{BB}+\mathrm{ACEI} / \mathrm{ARB}+\mathrm{S}$ & $\begin{array}{l}\mathrm{AP}+\mathrm{ACEI} / \\
\mathrm{ARB}+\mathrm{S}\end{array}$ & $\mathrm{AP}+\mathrm{BB}+\mathrm{ACEI} / \mathrm{ARB}+\mathrm{S}$ \\
\hline Amar et al. [35] & 2008 & $\begin{array}{l}88.5 \text { at discharge } \\
83.8 \text { at } 14 \text { months }\end{array}$ & $\begin{array}{l}53.5 \text { at discharge } \\
44.8 \text { at } 14 \text { months }\end{array}$ & & $\begin{array}{l}74.5 \text { at discharge } \\
65.9 \text { at } 14 \text { months }\end{array}$ & & & $\begin{array}{l}46.2 \% \text { at discharge } \\
45.6 \% \text { at } 14 \text { months of } \\
\text { post-discharge }\end{array}$ \\
\hline Lee et al. [41] & 2008 & & & & & $\begin{array}{l}29.9 \% \text { at } 3 \text { months of post-discharge } \\
37.5 \% \text { at } 6 \text { months of post-discharge } \\
42.6 \% \text { at } 12 \text { months of post-discharge } \\
46.3 \% \text { at } 18 \text { months of post-discharge }\end{array}$ & & \\
\hline Bi et al. [27] & 2009 & $\begin{array}{l}78.5 \text { at discharge } \\
63.8 \text { at } 6 \text { months } \\
57.5 \text { at } 12 \text { months }\end{array}$ & & & & & & $\begin{array}{l}47.7 \% \text { at discharge } \\
43.6 \% \text { at } 6 \text { months } \\
40.6 \% \text { at } 12 \text { months }\end{array}$ \\
\hline $\begin{array}{l}\text { Wong et al. [37] } \\
\text { Javed et al. [33] }\end{array}$ & $\begin{array}{l}2009 \\
2011\end{array}$ & & & & $22.7 \%$ at discharge & & & $\begin{array}{l}25.1 \% \text { at discharge } \\
57 \% \text { at discharge } \\
48 \% \text { at } 3 \text { months of post-discharge }\end{array}$ \\
\hline $\begin{array}{l}\text { Bourdès } \\
\text { et al. [50] } \\
\text { Andrikopoulos } \\
\text { et al. [30] }\end{array}$ & $\begin{array}{l}2011 \\
2012\end{array}$ & $\begin{array}{l}97.6 \% \text { at } \\
\text { discharge }\end{array}$ & & & $\begin{array}{l}90.9 \% \text { at discharge } \\
93.2 \text { at discharge } \\
87.7 \text { at } 6 \text {-month } \\
\text { follow-up }\end{array}$ & & $\begin{array}{l}92.4 \% \text { at } \\
\text { discharge }\end{array}$ & $86.7 \%$ at discharge \\
\hline $\begin{array}{l}\text { Shimony } \\
\text { et al. [16] }\end{array}$ & 2014 & & & & & & $\begin{array}{l}58,3 \text { at } \\
\text { discharge }\end{array}$ & \\
\hline $\begin{array}{l}\text { Gausia } \\
\text { et al. [20] }\end{array}$ & 2014 & $2.6 \%$ at discharge & $0.6 \%$ at discharge & $\begin{array}{l}0.2 \% \text { at } \\
\text { discharge }\end{array}$ & $10.1 \%$ at discharge & $0.6 \%$ at discharge & $\begin{array}{l}10.5 \% \text { at } \\
\text { discharge }\end{array}$ & $50.7 \%$ at discharge \\
\hline Selby et al. [9] & 2015 & & & & & & & $\begin{array}{l}41,8 \text { at discharge } \\
51,05 \text { at } 30 \text { days of post-discharge }\end{array}$ \\
\hline
\end{tabular}


Table 4: The characteristics of studies

\begin{tabular}{|c|c|c|c|c|c|c|c|}
\hline Study & Year & Country & Time points & Data Source & Patients characteristics & Design & $\begin{array}{l}\text { Sample } \\
\text { Size }\end{array}$ \\
\hline Amar et al. [35] & 2008 & France & $\begin{array}{l}\text { At discharge to 14-month } \\
\text { follow-up }\end{array}$ & PREVENIR-4 study & $\begin{array}{l}\text { Patients hospitalized with } \\
\text { ACS (2005) }\end{array}$ & Cross-Sectional & 1700 \\
\hline Lee et al. [41] & 2008 & US (Mid Atlantic state) & $\begin{array}{l}3,6,12 \text {, and } 18 \text {-months } \\
\text { follow-up }\end{array}$ & $\begin{array}{l}\text { Medical claim from } \\
\text { Managed Care } \\
\text { Organization }\end{array}$ & Patients with ACS at discharge & Cohort & 1135 \\
\hline $\begin{array}{l}\text { Vermeer and } \\
\text { Bajorek [13] }\end{array}$ & 2008 & Australia & At discharge & $\begin{array}{l}1 \text { Major public teaching } \\
\text { hospital }\end{array}$ & $\begin{array}{l}\text { Patients diagnosed as primary or } \\
\text { secondary ACS (January-April 2007) }\end{array}$ & Cross-Sectional & 169 \\
\hline Bi et al. [27] & 2009 & China & $\begin{array}{l}\text { At discharge, } 6 \text { and } \\
\text { 12-months follow-up }\end{array}$ & $\begin{array}{l}51 \text { Hospitals (Secondary } \\
\text { and Tertiary Hospital) }\end{array}$ & $\begin{array}{l}\text { Patients admitted to hospitals with a } \\
\text { diagnosis of STEMI, NSEMI, or UA } \\
\text { during Sept 2004-May } 2006\end{array}$ & Cohort & 2901 \\
\hline Wong et al. [37] & 2009 & New Zealand & At discharge & 2 Coronary Care Units & $\begin{array}{l}\text { Hospital survivors with ACS } \\
\text { discharged during 2000-2002 }\end{array}$ & Cohort & 1057 \\
\hline Wong et al. [8] & 2009 & New Zealand & $\begin{array}{l}\text { At discharge to } 5 \text {-year } \\
\text { follow-up }\end{array}$ & 2 Coronary Care Units & $\begin{array}{l}\text { Hospital survivors with ACS } \\
\text { discharged during } 2000-2002 \\
\text { prescribed with aspirin }\end{array}$ & Cohort & 1025 \\
\hline Abdallah et al. [14] & 2010 & Lebanon & $\begin{array}{l}\text { In hospital and at } \\
\text { discharge }\end{array}$ & $\begin{array}{l}\text { Tertiary referral university } \\
\text { hospital }\end{array}$ & $\begin{array}{l}\text { Patients hospitalized and diagnosed } \\
\text { with ACS (2002-2005) }\end{array}$ & Cross-Sectional & 1025 \\
\hline Melloni et al. [28] & 2010 & USA & $\begin{array}{l}\text { At admission, at } \\
\text { discharge, and 12-month } \\
\text { follow-up }\end{array}$ & MAINTAIN Registry & $\begin{array}{l}\text { ACS patients (January } \\
\text { 2006-September 2007) }\end{array}$ & Cohort & 788 \\
\hline Ramanath et al. [17] & 2010 & USA & $\begin{array}{l}\text { In a hospital, at discharge } \\
\text { and 6-month follow-up }\end{array}$ & $\begin{array}{l}\text { University of Michigan } \\
\text { Health System's ACS } \\
\text { registry }\end{array}$ & $\begin{array}{l}\text { Patients hospitalized due to } \\
\text { ACS and underwent coronary } \\
\text { angiography }\end{array}$ & Cohort & 2264 \\
\hline $\begin{array}{l}\text { Aliprandi-Costa } \\
\text { et al. [18] }\end{array}$ & 2011 & $\begin{array}{l}\text { Australia } \\
\text { New Zealand }\end{array}$ & $\begin{array}{l}\text { In-hospital and 6-month } \\
\text { follow-up }\end{array}$ & GRACE registry & 17,263 STEMI and 3892 NSTE-ACS & Cohort & 5615 \\
\hline Bourdès et al. [50] & 2011 & France & At discharge & PREVENIR-5 study & $\begin{array}{l}\text { Patients hospitalized for the } 1^{\text {st }} \\
\text { episode of ACS }\end{array}$ & Cross-Sectional & 4850 \\
\hline Javed et al. [33] & 2011 & USA & At discharge & GWTG program & $\begin{array}{l}\text { ACS related hospitalization from } \\
\text { 2005-2009 }\end{array}$ & Cohort & 159713 \\
\hline $\begin{array}{l}\text { The Access } \\
\text { Investigators [29] }\end{array}$ & 2011 & $\begin{array}{l}\text { Africa } \\
\text { Latin America } \\
\text { Middle Eastern Countries }\end{array}$ & $\begin{array}{l}\text { At admission, at } \\
\text { discharge, } 6 \text { and } \\
12 \text {-months follow-up }\end{array}$ & ACCESS registry & $\begin{array}{l}\text { Patients hospitalized with } \\
\text { ACS (2007-2008) 46.1\%STEMI and } \\
54 \% \text { NSTE-ACS }\end{array}$ & Cohort & 11731 \\
\hline $\begin{array}{l}\text { Andrikopoulos } \\
\text { et al. [30] }\end{array}$ & 2012 & Greece & $\begin{array}{l}\text { At discharge and } \\
\text { 6-month follow-up }\end{array}$ & $\begin{array}{l}\text { TARGET study ( } 17 \\
\text { centers) }\end{array}$ & $\begin{array}{l}\text { Patients admitted with ACS (2012): } \\
44.7 \% \text { STEMI, } 34.2 \% \text { NSTEMI, } \\
21.1 \% \text { UA }\end{array}$ & Cohort & 418 \\
\hline Kim et al. [42] & 2012 & Korea & $\begin{array}{l}\text { In-hospital and 30-day } \\
\text { follow-up }\end{array}$ & MUSTANG Registry & $\begin{array}{l}\text { Patients presented with } \mathrm{ACS} \text { and } \\
\text { underwent } \mathrm{PCl}\end{array}$ & Cohort & 3362 \\
\hline Ranasinghe et al. [19] & 2012 & $\begin{array}{l}\text { Australia } \\
\text { New Zealand }\end{array}$ & $\begin{array}{l}\text { In a hospital, at } \\
\text { discharge, and 6-month } \\
\text { follow-up }\end{array}$ & GRACE registry & $\begin{array}{l}\text { Patients hospitalized and diagnosed } \\
\text { with ACS at admission and } \\
\text { discharge time points }\end{array}$ & Cohort & 5556 \\
\hline Wai et al. [36] & 2012 & Australia & $\begin{array}{l}\text { At discharge, } 14 \text {-day and } \\
\text { 3-month follow-up }\end{array}$ & $\begin{array}{l}\text { DMACS project ( } 49 \\
\text { hospitals) }\end{array}$ & $\begin{array}{l}\text { Patients discharged with } \\
\text { ACS (June-Sep 2008) 22\%STEMI, } \\
38 \% \text { NSTEMI, 20\% UA, 20\% } \\
\text { Un-specified }\end{array}$ & Cross Sectional & 1545 \\
\hline Yusuf et al. [7] & 2012 & USA & $\begin{array}{l}\text { At discharge and } \\
\text { 12-month follow-up }\end{array}$ & 1 University hospital & $\begin{array}{l}\text { Patients discharged with acute } \\
\text { MI (2000-2006) }\end{array}$ & Cohort & 456 \\
\hline $\begin{array}{l}\text { Andrikopoulos } \\
\text { et al. [30] }\end{array}$ & 2013 & Greece & $\begin{array}{l}\text { At discharge and } \\
6 \text {-month follow-up }\end{array}$ & TARGET study & $\begin{array}{l}\text { Patients with ACS admitted to the } \\
\text { selected } 17 \text { hospitals }\end{array}$ & Cohort & 366 \\
\hline Huffman et al. [55] & 2013 & India & $\begin{array}{l}\text { In hospital and at } \\
\text { discharge }\end{array}$ & Kerala ACS registry & $\begin{array}{l}\text { ACS patients admitted to } 125 \\
\text { hospitals (2007-2009) }\end{array}$ & Cross-Sectional & 25718 \\
\hline Kassab et al. [15] & 2013 & Malaysia & $\begin{array}{l}\text { At admission and } \\
\text { discharge }\end{array}$ & 1 Tertiary hospital & $\begin{array}{l}\text { Patients with a primary diagnosis } \\
\text { with ACS }\end{array}$ & Cross-Sectional & 380 \\
\hline Shehab et al. [6] & 2013 & $\begin{array}{l}6 \text { Middle Eastern Countries } \\
\text { Bahrain } \\
\text { Saudi Arabia } \\
\text { Qatar } \\
\text { Oman } \\
\text { United Arab Emirates } \\
\text { Yemen }\end{array}$ & $\begin{array}{l}\text { At admission, at } \\
\text { discharge, and } 12 \\
\text { months follow up }\end{array}$ & Gulf RACE-2 Registry & $\begin{array}{l}\text { Patients hospitalized with ACS } \\
\text { as final diagnostic from } 65 \\
\text { hospitals (2008-2009) }\end{array}$ & Cohort & 7930 \\
\hline Zeymer et al. [72] & 2013 & $\begin{array}{l}\text { Spain } \\
\text { UK } \\
\text { France } \\
\text { Czech rep } \\
\text { Germany } \\
\text { Greece } \\
\text { Norway } \\
\text { Austria } \\
\text { Hungary } \\
\text { Belgium } \\
\text { Netherland } \\
\text { Sweden } \\
\text { Denmark } \\
\text { Finland }\end{array}$ & $\begin{array}{l}\text { At admission, in hospital, } \\
\text { at discharge, 3-month, } \\
6 \text {-month, and 12-month } \\
\text { follow-up }\end{array}$ & APTOR registry & $\begin{array}{l}\text { Patients presented with ACS and } \\
\text { underwent } \mathrm{PCl}\end{array}$ & Cohort & 4546 \\
\hline Gausia et al. [20] & 2014 & Australia & $\begin{array}{l}\text { At admission and } \\
\text { discharge }\end{array}$ & $\begin{array}{l}\text { WA hospital morbidity } \\
\text { Data Linkage System }\end{array}$ & $\begin{array}{l}\text { ACS patients discharged } \\
\text { alive }(2002-2004)\end{array}$ & Cohort & 1717 \\
\hline Grey et al. [71] & 2014 & New Zealand & $\begin{array}{l}\text { At discharge, } 7 \text {-day, } \\
\text { 30-day, 90-day, } \\
\text { 12-month, 2-year, and } \\
\text { 3-year follow-up }\end{array}$ & $\begin{array}{l}\text { National datasets linkage } \\
\text { of Public Hospital }\end{array}$ & $\begin{array}{l}\text { ACS patients discharged from } \\
\text { hospital over the year in } 2007\end{array}$ & Cohort & 11348 \\
\hline Jin et al. [31] & 2014 & China & $\begin{array}{l}\text { At discharge and } \\
\text { 12-month follow-up }\end{array}$ & $\begin{array}{l}\text { Cardiac center unit at a } \\
\text { university hospital }\end{array}$ & $\begin{array}{l}\text { Hospitalized patients with } \\
\text { ACS (2009-2011) }\end{array}$ & Cohort & 469 \\
\hline Maggioni et al. [38] & 2014 & Italy & At discharge & $\begin{array}{l}\text { ARNO Observatory record } \\
\text { linkage ( } 7 \text { local Italian } \\
\text { health authorities) }\end{array}$ & Patients discharged with ACS & Cross-Sectional & 3078 \\
\hline Pereira et al. [54] & 2014 & Portugal & At discharge & 10 Public Hospitals & $\begin{array}{l}\text { Patients discharged with ACS (744 } \\
\text { STEMI and } 1364 \text { NSTE-ACS) }\end{array}$ & Cohort & 2111 \\
\hline
\end{tabular}


Table 4: (Continued)

\begin{tabular}{|c|c|c|c|c|c|c|c|}
\hline $\begin{array}{l}\text { Study } \\
\text { St }\end{array}$ & Year & Country & Time points & Data Source & Patients characteristics & Design & $\begin{array}{l}\text { Sample } \\
\text { Size }\end{array}$ \\
\hline Shimony et al. [16] & 2014 & $\begin{array}{l}\text { High-income (Canada and } \\
\text { United States) and Low/ } \\
\text { middle-income (India, Iran, } \\
\text { Pakistan, and Tunisia) }\end{array}$ & At discharge & $\begin{array}{l}\text { ZESCA study ( } 38 \text { Centers } \\
\text { from } 6 \text { countries) }\end{array}$ & $\begin{array}{l}\text { Current smoker (smoked } \geq 10 \\
\text { cigarettes/day) ACS patients } \\
\text { admitted to the ICCU or similar type } \\
\text { of cardiology ward }\end{array}$ & Cross-Sectional & $\begin{array}{l}392(265 \\
\text { from HIC, } \\
127 \text { from } \\
\text { LMIC) }\end{array}$ \\
\hline Wang et al. [51] & 2014 & Brazil & $\begin{array}{l}\text { In a hospital, at } \\
\text { discharge, and 6-month } \\
\text { follow-up }\end{array}$ & ACCEPT registry & ACS patients (2011-2012) & Cohort & 2453 \\
\hline Anzai et al. [23] & 2015 & Japan & $\begin{array}{l}\text { In a hospital, at } \\
\text { discharge, and 2-year } \\
\text { follow-up }\end{array}$ & 1 Teaching hospital & $\begin{array}{l}\text { Patients underwent } \mathrm{PCI} \text { for ACS with } \\
\text { stenting (2005-2009) }\end{array}$ & Cohort & 405 \\
\hline Gencer et al. [49] & 2015 & Switzerland & $\begin{array}{l}\text { At discharge and } \\
\text { 12-month follow-up }\end{array}$ & 4 Teaching hospitals & $\begin{array}{l}\text { ACS patients hospitalized during } \\
2009-2012\end{array}$ & Cohort & 1602 \\
\hline Ghadri et al. [21] & 2015 & Switzerland & $\begin{array}{l}\text { In-hospital and 30-day } \\
\text { follow-up }\end{array}$ & $\begin{array}{l}\text { Z-ACS registry ( } 1 \\
\text { university hospital) }\end{array}$ & $\begin{array}{l}\text { ACS patients underwent coronary } \\
\text { angiography during } 2007-2012\end{array}$ & Cohort & 2612 \\
\hline Kassaian et al. [32] & 2015 & Iran & $\begin{array}{l}1 \text { month and } 12 \text {-month } \\
\text { follow-up post-discharge }\end{array}$ & 11 Tertiary hospitals & $\begin{array}{l}\text { Patients discharged alive with } \\
\text { confirmed ACS }\end{array}$ & Cohort & 1799 \\
\hline Medagama et al. [22] & 2015 & Sri Lanka & $\begin{array}{l}\text { In hospital and at } \\
\text { discharge }\end{array}$ & 1 Tertiary teaching hospital & $\begin{array}{l}\text { Patients presented with } \\
\text { ACS (November 2011-March 2012) }\end{array}$ & Cohort & 256 \\
\hline Selby et al. [9] & 2015 & Switzerland & At admission & Teaching hospital & $\begin{array}{l}\text { Patients admitted with ACS without } \\
\text { previous CVD }\end{array}$ & Cross-Sectional & 3172 \\
\hline $\begin{array}{l}\text { Ferreira-González } \\
\text { et al. [44] }\end{array}$ & 2016 & Spain & $\begin{array}{l}\text { At discharge and 2-year } \\
\text { follow-up }\end{array}$ & $\begin{array}{l}\text { ACDC registry ( } 22 \\
\text { hospitals) }\end{array}$ & $\begin{array}{l}\text { Patients admitted with } \\
\text { ACS + PCI (Jan-April 2008) }\end{array}$ & Cohort & 917 \\
\hline Gunnell et al. [39] & 2016 & Western Australia & $\begin{array}{l}\text { At discharge and } \\
20 \text { years follow-up }\end{array}$ & $\begin{array}{l}\text { Western Australia Data } \\
\text { Linkage System }\end{array}$ & Patients alive after ACS (2008) & Cohort & 23642 \\
\hline Boccara et al. [24] & 2017 & France & $\begin{array}{l}1 \text { month, 6-month, } \\
12 \text {-month, 18-month, } \\
2 \text {-year, and 3-year } \\
\text { follow-up }\end{array}$ & PACS-HIV study & $\begin{array}{l}\text { Post-hospital discharged patients } \\
\text { with ACS and received statin } \\
\text { prescription (2003-2006) }\end{array}$ & Cohort & 282 \\
\hline Eisen et al. [10] & 2017 & $\begin{array}{l}36 \text { countries from North } \\
\text { America, South America, } \\
\text { Western Europe, Eastern } \\
\text { Europe, Asia Pacific) }\end{array}$ & $\begin{array}{l}\text { 3-month and 6-month } \\
\text { follow-up post-discharge }\end{array}$ & SOLID-TIMI 52 study & Patients after ACS (2009-2011) & Cohort & 12446 \\
\hline Khedri et al. [40] & 2017 & Sweden & $\begin{array}{l}\text { At admission, at } \\
\text { discharge, and 3-month } \\
\text { follow-up }\end{array}$ & $\begin{array}{l}\text { SWEDEHEART } \\
\text { registry ( } 72 \text { hospitals) }\end{array}$ & $\begin{array}{l}\text { Patients admitted with first } \\
\text { ACS (2005-2010) }\end{array}$ & Cohort & 77432 \\
\hline Mantel et al. [45] & 2017 & Sweden & $\begin{array}{l}\text { 12-month follow-up } \\
\text { post-discharge }\end{array}$ & $\begin{array}{l}\text { National Population-based } \\
\text { data linkage }\end{array}$ & $\begin{array}{l}\text { Patients experienced first MI or } \\
\text { UA (2007-2010) }\end{array}$ & Cohort & 4319 \\
\hline Turner et al. [46] & 2017 & UK & $\begin{array}{l}\text { At discharge, } 1 \text { month } \\
\text { and } 12 \text {-month follow-up }\end{array}$ & $\begin{array}{l}\text { PhACS study, NSTE-ACS } \\
\text { cohort }\end{array}$ & $\begin{array}{l}\text { ACS patients discharged on high } \\
\text { potency statin }\end{array}$ & Cohort & 1005 \\
\hline Al-Zakwani et al. [47] & 2018 & 4 Middle Eastern Countries & $\begin{array}{l}\text { At admission, in hospital, } \\
\text { at discharge, } 1 \text { month, } \\
6 \text {-month, and } 12 \text {-month } \\
\text { follow up }\end{array}$ & $\begin{array}{l}\text { Gulf COAST registry ( } 24 \\
\text { hospitals) }\end{array}$ & $\begin{array}{l}\text { Patients diagnosed with ACS } \\
\text { admitted to the hospital (2012-2013) }\end{array}$ & Cohort & 3681 \\
\hline Boklage et al. [26] & 2018 & USA & $\begin{array}{l}\text { At admission, in-hospital } \\
\text { and } 12 \text {-month follow-up }\end{array}$ & $\begin{array}{l}\text { MarketScan Research } \\
\text { Databases }\end{array}$ & $\begin{array}{l}\text { Patients who experienced at least } \\
1 \text { inpatient admission with ACS as } \\
\text { primary diagnose (2002-2014) }\end{array}$ & Cohort & 7802 \\
\hline $\begin{array}{l}\text { Hoedemaker } \\
\text { et al. [25] }\end{array}$ & 2018 & Netherland & $\begin{array}{l}\text { In a hospital, 30-day and } \\
12 \text {-month follow-up }\end{array}$ & $\begin{array}{l}1 \text { Tertiary hospital (Single } \\
\text { center registry) }\end{array}$ & $\begin{array}{l}\text { STEMI and NSTEMI patients } \\
\text { admitted to a hospital (2006-2014) }\end{array}$ & Cohort & 9202 \\
\hline Sun et al. [48] & 2018 & China & $\begin{array}{l}\text { 6-month and 12-month } \\
\text { follow-up post-discharge }\end{array}$ & $\begin{array}{l}\text { CAPSC- } 2 \text { and CAPSC- } 3 \\
\text { registry }\end{array}$ & $\mathrm{ACS}+\mathrm{LDL}-\mathrm{c}<70 \mathrm{mg} / \mathrm{dl}$ & Cohort & 3374 \\
\hline Hao et al. [34] & 2019 & China & $\begin{array}{l}\text { In hospital and at } \\
\text { discharge }\end{array}$ & CCC-ACS registry & $\begin{array}{l}\text { Patients with STEMI or NSTE-ACS } \\
\text { at hospital discharge (2014-2018) }\end{array}$ & Cohort & 82196 \\
\hline Desta et al. [52] & 2020 & Ethiopia & $\begin{array}{l}\text { In hospital and at } \\
\text { discharge }\end{array}$ & 1 Specialized Hospital & $\begin{array}{l}\text { ACS patients admitted during } \\
2013-2018 \text { ( } 72.8 \% \text { STEMI, } 15.2 \% \\
\text { NSTEMI, } 12 \% \text { UA) }\end{array}$ & Cross-Sectional & 151 \\
\hline
\end{tabular}

\section{Discussion}

The present systematic review included data regarding statin utilization from the 49 studies over the world. Our review found that the rate of statin utilization at discharge varied from $20 \%$ to $99 \%$. It should be noted that one-third (ten studies) of the included studies, which reported the use of statin at discharge, found that less than $80 \%$ of ACS patients received statin at hospital discharge. It should be noted that almost all those studies [7], [8], [14], [20], [23], [24], [33], [37], [55] collected the data before 2013 except Boccara et al. [24], who collected the data from 2002 to 2014 when the recommendation of using statin as primary prevention and secondary prevention for ACS was just published in 2014 [4].

About $64 \%$ of the studies found that statin utilization rate during hospitalization was higher than $80 \%$. Of the four studies, which reported statin utilization rate during less than $80 \%$ hospitalization, two studies were conducted in low and middle-income countries, including Lebanon [14] and Ethiopia [52]. The affordability and limited access to the essential medicines were reported among the low- and middleincome countries [56]. The others were conducted in high-income countries, but they used retrospective data in 1999-2007 [19] and 2002-2014 [26].

Although existing evidence indicated that adherence to statin treatment was associated with the reduction in cardiovascular related events and allcause mortality [57], [58], [59], a previous systematic review found a low adherence rate of statin treatment [60]. Similarly, almost all included studies in our review, which examined the statin utilization trend along the follow-up time points, found that the level of statin use was diminished since the discharge time point. It could probably be due to several reasons, including the 


\section{Table 5: Statin utilization and pattern of staying usage}

\begin{tabular}{|c|c|c|c|c|c|c|}
\hline \multirow[t]{2}{*}{ Study } & \multicolumn{4}{|l|}{ Statin utilizationxc } & \multirow[t]{2}{*}{ Pattern of statin use } & \multirow[t]{2}{*}{ Factor predicting statin use } \\
\hline & At Admission (\%) & In Hospital (\%) & At Discharge (\%) & Post Discharge (\%) & & \\
\hline Amar et al. [35] & & & 89.2 & $85.6(14 \mathrm{mos})$ & $\begin{array}{l}46.2 \%, 45.6 \% \text { use } \\
\text { combination of } 4 \\
\text { treatments (Beta blocker, } \\
\text { antiplatelet, stain, ACE) } \\
\text { at discharge and } 14 \text { mos } \\
\text { follow-up }\end{array}$ & \\
\hline Lee et al. [41] & & & & $\begin{array}{l}62.6 \text { (3 mos) } \\
60.3 \text { (6 mos }) \\
73.5 \text { (12 mos) } \\
76.6 \text { (18 mos) }\end{array}$ & & $\begin{array}{l}\text { Older patients were less } \\
\text { likely to receive statin } \\
(p<0.001) \\
\text { Women were less likely } \\
\text { than men to receive } \\
\text { statin }(<0.001)\end{array}$ \\
\hline $\begin{array}{l}\text { Vermeer and } \\
\text { Bajorek [13] }\end{array}$ & 40 & & 85 & 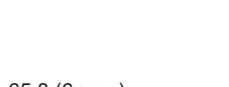 & & $\begin{array}{l}\text { Men were likely to be } \\
\text { discharged with a statin; } \\
\text { OR = } 3.36(1.11,10.15)\end{array}$ \\
\hline Bi et al. [27] & & & 80.4 & $\begin{array}{l}65.8 \text { (6 mos) } \\
59.4 \text { (12 mos })\end{array}$ & & \\
\hline $\begin{array}{l}\text { Wong et al. [8] } \\
\text { Abdallah et al. [14] }\end{array}$ & & $\begin{array}{l}59(62 \% \text { in STEMI, } \\
53 \% \text { in NSTEMI, } \\
62 \% \text { in UA, } p=0.03)\end{array}$ & $\begin{array}{l}58.8 \text { ( } 47 \% \text { for patients without } \\
\text { revascularization; } 73 \% \text { among } \\
\text { patients with revascularization) } \\
59.5 \\
60(64 \% \text { for STEMI, } 51 \% \text { for } \\
\text { NSTEMI, } 64 \% \text { for UA, p < } 0.01 \text { ) }\end{array}$ & & & \\
\hline $\begin{array}{l}\text { Melloni et al. [28] } \\
\text { Ramanath et al. [17] }\end{array}$ & 40 & & $\begin{array}{l}89.5 \\
80.6 \text { (69.1\% among } \\
\text { non-obstructive CAD, } 81.1 \% \\
\text { among obstructive CAD) }\end{array}$ & 66.7 (12 mos) & & \\
\hline $\begin{array}{l}\text { Aliprandi-Costa } \\
\text { et al. [18] }\end{array}$ & & & $\begin{array}{l}\text { 64.5, } 65.4 \text { for STEMI, } \\
\text { NSTEACS (2000-2001) } \\
\text { 80, 80.6 for STEMI, } \\
\text { NSTEACS (2004-2005) } \\
\text { 88.5, } 84.4 \text { for STEMI, } \\
\text { NSTEACS (2006-2007) }\end{array}$ & & & \\
\hline Bourdès et al. [50] & & & & $\begin{array}{l}\text { Of } 2131 \text { patients who } \\
\text { received EBCM at } \\
\text { discharge, } 98.1 \% \\
\text { still used statin at } 24 \\
\text { months after discharge }\end{array}$ & & \\
\hline Javed et al. [33] & & & $\begin{array}{l}\text { The use of intensive statin } \\
\text { monotherapy: } 26.9 \text { at } 2005 \\
29.1 \text { at } 2006 \\
30.2 \text { at } 2007 \\
30.4 \text { at } 2008 \\
32.2 \text { at } 2009\end{array}$ & & & \\
\hline $\begin{array}{l}\text { The Access of } \\
\text { Investigators (29) }\end{array}$ & & $\begin{array}{l}90.7 \text { ( } 90 \% \text { in } \\
\text { NSTE-ACS; } 91 \% \text { in } \\
\text { STEMI) }\end{array}$ & $\begin{array}{l}89.2(88 \% \text { in NSTE-ACS; } 91 \% \\
\text { in STEMI) }\end{array}$ & & & \\
\hline $\begin{array}{l}\text { Andrikopoulos } \\
\text { et al. [30] }\end{array}$ & 40 & 96 & 93 & & & \\
\hline $\begin{array}{l}\text { Kim et al. [42] } \\
\text { Ranasinghe et al. [19] }\end{array}$ & 49.8 & 76 & 83.7 & & & \\
\hline $\begin{array}{l}\text { Wai et al. [36] } \\
\text { Yusuf et al. [7] }\end{array}$ & & & $\begin{array}{l}92 \\
20.6\end{array}$ & 89 (3 mos) & & \\
\hline $\begin{array}{l}\text { Andrikopoulos } \\
\text { et al. [53] }\end{array}$ & & & 93.2 & 87.7 (6 mos) & & \\
\hline Huffman et al. [55] & 11.43 & & 78.9 & & & $\begin{array}{l}\text { In hospital: } 40 \% \text { received } \\
\text { optimal treatment (Aspirin, } \\
\text { clopidogrel, Beta-blocker, } \\
\text { statin, and heparin) } \\
\text { At discharge: } 46 \% \text { received } \\
\text { optimal treatment (Aspirin, } \\
\text { clopidogrel, Beta-blocker, } \\
\text { statin) }\end{array}$ \\
\hline Kassab et al. [15] & & & 95.9 & & & \\
\hline Shehab et al. [6] & $\begin{array}{l}94.8 \text { (Male } 95.1 \% \\
\text { female } 93.6 \%, p= \\
0.019)\end{array}$ & & $\begin{array}{l}91(\text { Male }=92.1 \%, \text { female }= \\
88.2 \%, p<0.001)\end{array}$ & & & $\begin{array}{l}\text { Female are less likely than } \\
\text { male to received statin } \\
\text { during hospitalization and } \\
\text { at discharge }\end{array}$ \\
\hline Zeymer et al. [72] & 34 & & 89 & $\begin{array}{l}88.5(6 \mathrm{mos}) \\
87(12 \mathrm{mos})\end{array}$ & & \\
\hline Gausia et al. [20] & & & $\begin{array}{l}75.4 \% \text { (aboriginal } 73.5 \% \text {, } \\
\text { non-aboriginal } 76.2 \%, p=0.25 \text { ) }\end{array}$ & & & \\
\hline Grey et al. [71] & 44 & & & $\begin{array}{l}59 \text { ( } 7 \text { days }) \\
71 \text { ( } 30 \text { days }) \\
83 \text { ( } 3 \text { mos }) \\
69 \text { ( } 12 \text { mos }) \\
67 \text { ( } 36 \text { mos })\end{array}$ & & \\
\hline Jin et al. [31] & & & $\begin{array}{l}88.8 \text { ( } 85.1 \text { in elderly vs. } 90.6 \text { in } \\
\text { non-elderly, } p=0.067 \text { ) }\end{array}$ & $\begin{array}{l}24.7 \text { ( } 12 \text { mos })(21.8 \\
\text { in elderly vs. } 29.6 \text { in } \\
\text { non-elderly, } 9=0.005)\end{array}$ & & $\begin{array}{l}\text { Underused at follow-up } \\
\text { occurred in elderly > } \\
\text { non-elderly }\end{array}$ \\
\hline Maggioni et al. [38] & & & 80.3 & $67.2(12 \mathrm{mos})$ & $\begin{array}{l}\text { At discharge: } 55 \% \\
\text { received atorvastatin, } \\
26.6 \% \text {-simvastatin, } \\
14.8 \% \text {-rosuvastatin, } \\
10.1 \% \text {-pravastatin, } \\
4.8 \% \text {-Fluvastatin, } \\
\text { 8.5\%-Simvastain+Ezetimibe } \\
0.6 \% \text {-Lovastatin }\end{array}$ & \\
\hline
\end{tabular}


Table 5: (Continued)

\begin{tabular}{|c|c|c|c|c|c|c|}
\hline \multirow[t]{2}{*}{ Study } & \multicolumn{4}{|l|}{ Statin utilizationxc } & \multirow[t]{2}{*}{ Pattern of statin use } & \multirow[t]{2}{*}{ Factor predicting statin use } \\
\hline & At Admission (\%) & In Hospital (\%) & At Discharge (\%) & Post Discharge (\%) & & \\
\hline Pereira et al. [54] & & & $\begin{array}{l}\text { 93\% among STEMI, } 90 \% \\
\text { among NSTE-ACS }\end{array}$ & & & $\begin{array}{l}\text { Patients aged } \geq 80 \text { years } \\
\text { with NSTE-ACS were } \\
\text { much less likely to } \\
\text { be discharged with } \\
\text { statins (OR } 0.35,95 \% \mathrm{Cl} \\
0.19-0.64 \text { ) }\end{array}$ \\
\hline Shimony et al. [16] & & & $\begin{array}{l}90.3 \% \text { in HIC } \\
76.8 \% \text { in LIC } \\
(\mathrm{OR}=2.8,95 \% \text { Cl: } 1.6-5.0)\end{array}$ & & & \\
\hline Wang et al. [51] & & 90.6 & 93 & 85.4 (6 mos) & & \\
\hline Anzai et al. [23] & & $\begin{array}{l}87 \text { (age }<80 \text { yrs) } \\
69 \text { (age } \geq 80 \text { yrs) }\end{array}$ & $\begin{array}{l}87 \text { (age }<80 \text { yrs) } \\
69 \text { (age } \geq 80 \text { yrs) }\end{array}$ & $\begin{array}{l}86 \text { (age }<80 \text { yrs) } \\
65 \text { (age } \geq 80 \text { yrs) }\end{array}$ & & The elderly were less likely \\
\hline Gencer et al. [49] & & & $\begin{array}{l}99 \text { (of this } 70 \text { were at } \\
\text { high-intensity statin) }\end{array}$ & 94 (12 mos) & & \\
\hline Ghadri et al. [21] & $\begin{array}{l}31.3 \text { (31.8 in male } \\
\text { vs. } 29.4 \text { in female, } p \\
=0.26)\end{array}$ & & $\begin{array}{l}88.5 \text { ( } 89.4 \text { in male vs. } 85.2 \text { in } \\
\text { female, } p=0.004)\end{array}$ & & & $\begin{array}{l}\text { Females were less likely } \\
\text { to receive a statin at } \\
\text { discharge as compared } \\
\text { to males }\end{array}$ \\
\hline Kassaian et al. [32] & & 94.3 & & & & \\
\hline Medagama et al. [22] & & 96.1 & 96.1 & & & \\
\hline Selby et al. [9] & $\begin{array}{l}16 \text { compared to } 27 \\
\text { eligible for statin }\end{array}$ & & & & & \\
\hline $\begin{array}{l}\text { Ferreira-González } \\
\text { et al. [44] }\end{array}$ & & & 89.4 & & & \\
\hline Gunnell et al. [39] & & & $\begin{array}{l}79.6(82 \% \text { in male, } 75.5 \% \text { in } \\
\text { female) }\end{array}$ & & & $\begin{array}{l}\text { Female were less likely to } \\
\text { dispense with a statin (OR } \\
=0.82 ; 95 \% \mathrm{Cl} 0.76-0.88)\end{array}$ \\
\hline Boccara et al. [24] & & & 12,4 & & & \\
\hline Eisen et al. [10] & & & 95.2 & & $\begin{array}{l}\text { Of those received statin, } \\
41.9 \% \text { got high intensity } \\
\text { statin. Of these patients, } \\
82 \% \text { were still on high } \\
\text { potency statin after } \\
2.3 \text { years }\end{array}$ & \\
\hline Khedri et al. [40] & 21 & & 84.4 & & & $\begin{array}{l}\text { Patients with eGFR } 30-59 \\
\text { were more likely to statin } \\
\text { treatment cessation } \\
(\mathrm{OR}=1.35,1.29-1.41)\end{array}$ \\
\hline Mantel et al. [45] & & & & $\begin{array}{l}73.5 \text { ( } 3 \mathrm{mos}) \\
63.5 \text { (6-12 mos })\end{array}$ & & \\
\hline Turner et al. [46] & & & & 84.4 (12 mos) & & \\
\hline Al-Zakwani et al. [47] & 61 & & 97 & & & \\
\hline Boklage et al. [26] & 30.5 & 70.9 & & 63.5 (12 mos) & & \\
\hline Hoedemaker et al. [25] & & & 85.2 & $\begin{array}{l}88.1 \text { (30 days) } \\
84.1 \text { ( } 12 \text { mos })\end{array}$ & & $\begin{array}{l}43.7,46.6,25.5 \text { received } \\
\text { optimal treatment at } \\
\text { discharge, } 30 \text { days, and } 12 \\
\text { mos, respectively. }\end{array}$ \\
\hline Sun et al. [48] & & & 85 & & & \\
\hline Hao et al. [34] & 17.5 & $\begin{array}{l}95.1 \text { (95.4 in male, } \\
94.3 \text { in female) }\end{array}$ & $\begin{array}{l}92.6 \text { ( } 93.2 \text { in male, } 90.7 \text { in } \\
\text { female) }\end{array}$ & & & $\begin{array}{l}\text { Female were less likely } \\
\text { to receive statin at } \\
\text { discharge }(O R=0.86 \text {. } \\
0.81-0.92)\end{array}$ \\
\hline Desta et al. [52] & & 84.1 & 94.7 & & & \\
\hline
\end{tabular}

side-effect of stain [61], [62], poor prescriber-patient relationship [60], and the quantity of received drugs at discharge [31]. The previous studies also found that under-used of statin among ACS was also associated with low education (OR 3.39; 95\% Cl 1.65-9.32), the greater number of comorbidities (OR 1.64; 95\% Cl 1.12-2.39), the quantity of received drugs at discharge (OR 1.31; 95\%Cl 1.11-1.55), low income (OR 3.97; $95 \% \mathrm{Cl} 1.47-10.75)$, and depression (OR 2.62; $95 \% \mathrm{Cl}$ 2.03-3.38) [31]. As the rate of statin utilization during follow-up was decreasing, effective intervention by a multi-disciplinary team, which included physician/ cardiologist, pharmacist as well as patient's family support to improve statin utilization among ACS should be implemented. Health system and policy support were also required to improve ACS evidence-based medicine adherence, including statin.

Our studies also revealed that statin utilization rate was lower among females, as compared to males. It could lead to higher mortality among female patients with ACS [63], [64], [65]. On the other hand, it could probably be due to the fact that males experienced more invasive procedures than females; thus, they were supposed to receive more statin prescriptions [6], [39]. Furthermore, statin utilization was also found to be lower among the elderly. A prior study reported that the number of concurrent medication and the comorbid diseases owned by the elderly could impact their adherence [31]. Therefore, more efforts should be made to improve the utilization rate among these patients.

This review is not without any limitations. First, only one database (PubMed) was used to identify studies. Second, our study mainly focused on statin utilization by putting aside other evidencebased treatment for ACS. However, recent guidelines recommended using statin among the ACS patients and recommended that high-risk statin be used among high-risk populations without considering their LDL-C level [2], [5], [66], [67]. It should be noted that our 
study did not mainly focus on the intensity of statin as well as other evidence-based treatment for secondary prevention among ACS. Nevertheless, our study could imply that the rate of evidence-based treatment among ACS patients would be even lower than the rate of statin utilization. Finally, it should be noted that the utilization rate of statin among ACS also depends on the characteristics of ACS patients, such as renal function [40], [68], liver function [69], and Parkinson's disease [70].

\section{Conclusion}

Although the benefits of statin in ACS patients have been established [6], [7], [8], [9], [10], our study revealed the under-utilization rate of statin for secondary prevention among ACS patients, especially during follow-up. This review highlighted the suboptimal adherence to the guideline recommendation in realworld practice. To improve patients' clinical outcomes with ACS, substantial efforts should be made to increase optimal treatment prescription among physicians and increase adherence of statin among ACS patients [5].

\section{References}

1. Ritchie MRaH. Burden of Disease; 2017. Available from: https:// www.ourworldindata.org/burden-of-disease. [Last accessed on 2020 Aug 11]

2. Mach F, Baigent C, Catapano AL, Koskinas KC, Casula M, Badimon L, et al. 2019 ESC/EAS Guidelines for the management of dyslipidaemias: Lipid modification to reduce cardiovascular risk. Eur Heart J. 2020;41(1):111-88. https://doi. org/10.15829/1560-4071-2020-3826

PMid:31504418

3. Ruscica M, Macchi C, Pavanello C, Corsini A, Sahebkar A, Sirtori CR. Appropriateness of statin prescription in the elderly. Eur J Intern Med. 2018;50:33-40. https://doi.org/10.1016/j. ejim.2017.12.011

PMid:29310996

4. Stone NJ, Robinson JG, Lichtenstein AH, Bairey Merz CN Blum CB, Eckel RH, et al. 2013 ACC/AHA guideline on the treatment of blood cholesterol to reduce atherosclerotic cardiovascular risk in adults: A report of the American College of Cardiology/American Heart Association Task Force on Practice Guidelines. J Am Coll Cardiol. 2014;63(25 Pt B):2889-934. https://doi.org/10.1161/01.cir.0000437738.63853.7a

PMid:24239923

5. ArnettDK, Blumenthal RS, Albert MA, Buroker AB, Goldberger ZD Hahn EJ, et al. 2019 ACC/AHA guideline on the primary prevention of cardiovascular disease: A report of the American College of Cardiology/American Heart Association Task Force on Clinical Practice Guidelines. Circulation. 2019;140(11):e596646. https://doi.org/10.1161/cir.0000000000000725

PMid:30879355
6. Shehab A, Al-Dabbagh B, AlHabib KF, Alsheikh-Ali AA, Almahmeed W, Sulaiman K, et al. Gender disparities in the presentation, management and outcomes of acute coronary syndrome patients: Data from the $2^{\text {nd }}$ Gulf Registry of Acute Coronary Events (Gulf RACE-2). PLoS One. 2013;8(2):e55508. https://doi.org/10.1371/journal.pone.0055508

PMid:23405162

7. Yusuf SW, Daraban N, Abbasi N, Lei X, Durand JB, Daher IN Treatment and outcomes of acute coronary syndrome in the cancer population. Clin Cardiol. 2012;35(7):443-50. https://doi. org/10.1002/clc.22007 PMid:22588943

8. Wong CK, Tang EW, Herbison P. Prognostic interactions between statins and in-hospital revascularisation on the outcome of acute coronary syndrome. Heart Lung Circ. 2009;18(4):262-5. https:// doi.org/10.1016/j.hlc.2008.12.001

PMid:19167924

9. Selby K, Nanchen D, Auer R, Gencer B, Räber L, Klingenberg R, et al. Low statin use in adults hospitalized with acute coronary syndrome. Prev Med. 2015;77:131-6. https://doi.org/10.1016/j. ypmed.2015.05.012

PMid:26007299

10. Eisen A, Cannon CP, Braunwald E, Steen DL, Zhou J, Goodrich EL, et al. Predictors of nonuse of a high-potency statin after an acute coronary syndrome: Insights from the stabilization of plaques using darapladib-thrombolysis in myocardial infarction 52 (SOLID-TIMI 52) trial. J Am Heart Assoc. 2017;6(1):e004332. https://doi.org/10.1161/jaha.116.004332

PMid:28077384

11. Downes MJ, Brennan ML, Williams HC, Dean RS. Development of a critical appraisal tool to assess the quality of cross-sectional studies (AXIS). BMJ Open. 2016;6(12):e011458. https://doi. org/10.1136/bmjopen-2016-011458 PMid:27932337

12. Peterson J, Welch V, Losos M, Tugwell P. The Newcastle-Ottawa Scale (NOS) for Assessing the Quality of Nonrandomised Studies in Meta-analyses. UK: University of Liverpool; 2000.

13. Vermeer NS, Bajorek BV. Utilization of evidence-based therapy for the secondary prevention of acute coronary syndromes in Australian practice. J Clin Pharm Ther. 2008;33(6):591-601. https://doi.org/10.1111/j.1365-2710.2008.00950.x PMid:19138236

14. Abdallah M, Karrowni W, Shamseddeen W, Itani S, Kobeissi L, Ghazzal Z, et al. Acute coronary syndromes: Clinical characteristics, management, and outcomes at the American University of Beirut Medical Center, 2002-2005. Clin Cardiol. 2010;33(1):E6-13. https://doi.org/10.1002/clc.20636 PMid:20014175

15. Kassab YW, Hassan $\mathrm{Y}$, Aziz NA, Akram $\mathrm{H}$, Ismail $\mathrm{O}$. Use of evidence-based therapy for the secondary prevention of acute coronary syndromes in Malaysian practice. J Eval Clin Pract. 2013;19(4):658-63. https://doi. org/10.1111/j.1365-2753.2012.01894.x PMid:22845427

16. Shimony A, Grandi SM, Pilote L, Joseph L, O'Loughlin J, Paradis $\mathrm{G}$, et al. Utilization of evidence-based therapy for acute coronary syndrome in high-income and low/middleincome countries. Am J Cardiol. 2014;113(5):793-7. https://doi. org/10.1016/j.amjcard.2013.11.024 PMid:24440324

17. Ramanath VS, Armstrong DF, Grzybowski M, RahnamaMohagdam S, Tamhane UU, Gordon K, et al. Receipt of cardiac medications upon discharge among men and women with acute coronary syndrome and nonobstructive coronary artery disease. Clin Cardiol. 2010;33(1):36-41. https://doi.org/10.1002/ 


\section{clc. 20701}

\section{PMid:20063300}

18. Aliprandi-Costa B, Ranasinghe I, Chow V, Kapila S, Juergens C Devlin G, et al. Management and outcomes of patients with acute coronary syndromes in Australia and New Zealand, 2000-2007. Med J Aust. 2011;195(3):116-21. https://doi. org/10.5694/j.1326-5377.2011.tb03237.x PMid:21806528

19. Ranasinghe I, Naoum C, Aliprandi-Costa B, Sindone AP, Steg PG, Elliott $\mathrm{J}$, et al. Management and outcomes following an acute coronary event in patients with chronic heart failure 1999-2007. Eur J Heart Fail. 2012;14(5):464-72. https://doi. org/10.1093/eurjhf/hfs041

\section{PMid:22499543}

20. Gausia K, Katzenellenbogen JM, Sanfilippo FM, Knuiman MW, Thompson PL, Hobbs MS, et al. Evidence-based prescribing of drugs for secondary prevention of acute coronary syndrome in Aboriginal and non-Aboriginal patients admitted to Western Australian hospitals. Intern Med J. 2014;44(4):353-61. https:// doi.org/10.1111/imj.12375

PMid:24528930

21. Ghadri JR, Sarcon A, Jaguszewski M, Diekmann J, Bataiosu RD, Hellermann J, et al. Gender disparities in acute coronary syndrome: A closing gap in the short-term outcome. J Cardiovasc Med (Hagerstown). 2015;16(5):355-62. https://doi. org/10.2459/jcm.0000000000000248

PMid:25826477

22. Medagama A, Bandara R, De Silva C, Galgomuwa MP. Management of acute coronary syndromes in a developing country; time for a paradigm shift? An observational study. BMC Cardiovasc Disord. 2015;15:133. https://doi.org/10.1186/ s12872-015-0125-y

PMid:26497226

23. Anzai A, Maekawa Y, Kodaira M, Mogi S, Arai T, Kawakami T, et al. Prognostic implications of optimal medical therapy in patients undergoing percutaneous coronary intervention for acute coronary syndrome in octogenarians. Heart Vessels. 2015;30(2):186-92. https://doi.org/10.1007/s00380-014-0474-y PMid:24493327

24. Boccara F, Miantezila Basilua J, Mary-Krause M, Lang S, Teiger E, Steg PG, et al. Statin therapy and low-density lipoprotein cholesterol reduction in HIV-infected individuals after acute coronary syndrome: Results from the PACS-HIV lipids substudy. Am Heart J. 2017;183:91-101. https://doi. org/10.1016/j.ahj.2016.10.013

PMid:27979047

25. Hoedemaker NP, Damman P, Ottervanger JP, Dambrink JH, Gosselink AT, Kedhi E, et al. Trends in optimal medical therapy prescription and mortality after admission for acute coronary syndrome: A 9-year experience in a real-world setting. Eur Heart J Cardiovasc Pharmacother. 2018;4(2):102-10. https:// doi.org/10.1093/ehjcvp/pvy005

\section{PMid:29394340}

26. Boklage SH, Malangone-Monaco E, Lopez-Gonzalez L, Ding Y Henriques C, Elassal J. Statin utilization patterns and outcomes for patients with acute coronary syndrome during and following inpatient admissions. Cardiovasc Drugs Ther. 2018;32(3):27380. https://doi.org/10.1007/s10557-018-6800-3 PMid:29855748

27. Bi Y, Gao R, Patel A, Su S, Gao W, Hu D, et al. Evidence-based medication use among Chinese patients with acute coronary syndromes at the time of hospital discharge and 1 year after hospitalization: Results from the Clinical Pathways for Acute Coronary Syndromes in China (CPACS) study. Am Heart J. 2009;157(3):509-16. https://doi.org/10.1016/j.ahj.2008.09.026

\section{PMid:19249422}

28. Melloni C, Shah BR, Ou FS, Roe MT, Smith SC Jr., Pollack CV Jr., et al. Lipid-lowering intensification and lowdensity lipoprotein cholesterol achievement from hospital admission to 1-year follow-up after an acute coronary syndrome event: Results from the Medications Applled aNd SusTAINed Over Time (MAINTAIN) registry. Am Heart J. 2010;160(6):11219. https://doi.org/10.1016/j.ahj.2010.09.008 PMid:21146667

29. ACCESS Investigators. Management of acute coronary syndromes in developing countries: Acute coronary events-a multinational survey of current management strategies. Am Heart J. 2011;162(5):852-9. https://doi.org/10.1016/j. ahj.2011.07.029 PMid:22093201

30. Andrikopoulos G, Tzeis S, Mantas I, Olympios C, Kitsiou A Kartalis A, et al. Epidemiological characteristics and in-hospital management of acute coronary syndrome patients in Greece: Results from the TARGET study. Hellenic J Cardiol. 2012;53(1):33-40. PMid:22275741

31. Jin $H$, Tang $C$, Wei $Q$, Chen $L$, Sun $Q$, Ma $G$, et al. Agerelated differences in factors associated with the underuse of recommended medications in acute coronary syndrome patients at leastone year after hospital discharge. BMC Cardiovasc Disord. 2014;14(1):127. https://doi.org/10.1186/1471-2261-14-127 PMid:25252927

32. Kassaian SE, Masoudkabir F, Sezavar H, Mohammadi M, Pourmoghaddas A, Kojuri J, et al. Clinical characteristics, management and 1-year outcomes of patients with acute coronary syndrome in Iran: The Iranian Project for Assessment of Coronary Events 2 (IPACE2). BMJ Open. 2015;5(12):e007786. https://doi.org/10.1136/bmjopen-2015-007786 PMid:26671947

33. Javed U, Deedwania PC, Bhatt DL, Cannon CP, Dai D, Hernandez AF, et al. Use of intensive lipid-lowering therapy in patients hospitalized with acute coronary syndrome: An analysis of 65,396 hospitalizations from 344 hospitals participating in Get With The Guidelines (GWTG). Am Heart J. 2010;160(6):1130-6. https://doi.org/10.1016/j.ahj.2010.08.041 PMid:21146668

34. Hao Y, Liu J, Liu J, Yang N, Smith SC, Jr., Huo $\mathrm{Y}$, et al. Sex differences in in-hospital management and outcomes of patients with acute coronary syndrome. Circulation. 2019;139(15):1776-85.

PMid:30667281

35. Amar J, Ferrieres J, Cambou JP, Amelineau E, Danchin N. Persistence of combination of evidence-based medical therapy in patients with acute coronary syndromes. Arch Cardiovasc Dis. 2008;101(5):301-6. https://doi.org/10.1016/j.acvd.2008.04.005 PMid: 18656088

36. Wai A, Pulver LK, Oliver K, Thompson A. Current discharge management of acute coronary syndromes: Baseline results from a national quality improvement initiative. Intern Med J. 2012;42(5):e53-9. https://doi. org/10.1111/j.1445-5994.2010.02308.x PMid:20646093

37. Wong CK, Tang EW, Herbison P. The use of different evidencebased medications and 5-year survival after an acute coronary syndrome:An observational study. Int J Cardiol. 2009;132(2):197202. https://doi.org/10.1016/j.ijcard.2007.11.005 PMid:18191242

38. Maggioni AP, Rossi E, Cinconze E, De Rosa M. Use and misuse of statins after ACS: Analysis of a prescription database of a community setting of 2,042,968 subjects. 
Eur J Prev Cardiol. 2014;21(9):1109-16. https://doi. org/10.1177/2047487313485511

PMid:23539716

39. Gunnell AS, Hung J, Knuiman MW, Nedkoff L, Gillies M, Geelhoed E, et al. Secondary preventive medication use in a prevalent population-based cohort of acute coronary syndrome survivors. Cardiovasc Ther. 2016;34(6):423-30. https://doi. org/10.1111/1755-5922.12212

PMid:27489053

40. Khedri M, Szummer K, Carrero JJ, Jernberg T, Evans M, Jacobson $\mathrm{SH}$, et al. Systematic underutilisation of secondary preventive drugs in patients with acute coronary syndrome and reduced renal function. Eur J Prev Cardiol. 2017;24(7):724-34. https://doi.org/10.1177/2047487317693950 PMid:28195517

41. Lee HY, Cooke CE, Robertson TA. Use of secondary prevention drug therapy in patients with acute coronary syndrome after hospital discharge. J Manag Care Pharm. 2008;14(3):271-80. https://doi.org/10.18553/jmcp.2008.14.3.271

PMid:18439049

42. Kim MJ, Jeon DS, Gwon HC, Kim SJ, Chang K, Kim HS, et al. Current statin usage for patients with acute coronary syndrome undergoing percutaneous coronary intervention: Multicenter survey in Korea. Clin Cardiol. 2012;35(11):700-6. https://doi. org/10.1002/clc.22038

PMid:22825844

43. Zeymer U, Berkenboom G, Coufal Z, Belger M, Sartral M, Norrbacka K, et al. Predictors, cost, and outcomes of patients with acute coronary syndrome who receive optimal secondary prevention therapy: Results from the antiplatelet treatment observational registries (APTOR). Int J Cardiol. 2013;170(2):23945. https://doi.org/10.1016/j.ijcard.2013.10.057

PMid:24225199

44. Ferreira-González I, Carrillo X, Martín V, de la Torre Hernández JM, Baz JA, Navarro Manchón J, et al. Interhospital variability in drug prescription after acute coronary syndrome: Insights from the ACDC study. Rev Esp Cardiol (Engl Ed). 2016;69(2):117-24. https://doi.org/10.1016/j.rec.2015.04.018 PMid:26253861

45. Mantel Ä, Holmqvist M, Jernberg T, Wållberg-Jonsson $\mathrm{S}$, Askling J. Long-term outcomes and secondary prevention after acute coronary events in patients with rheumatoid arthritis. Ann Rheum Dis. 2017;76(12):2017-24. https://doi.org/10.1136/ annrheumdis-2017-211608

PMid:28823986

46. Turner RM, Yin P, Hanson A, FitzGerald R, Morris AP, Stables $\mathrm{RH}$, et al. Investigating the prevalence, predictors, and prognosis of suboptimal statin use early after a non-ST elevation acute coronary syndrome. J Clin Lipidol. 2017;11(1):204-14. https://doi.org/10.1016/j.jacl.2016.12.007 PMid:28391887

47. Al-Zakwani I, Zubaid M, Alsheikh-Ali AA, Almahmeed W, Rashed W. Effect of evidence-based cardiac drug therapy on mortality in patients with acute coronary syndrome: Findings from the Gulf COAST registry. Cardiovasc Ther. 2018;36(6):e12463. https://doi.org/10.1111/1755-5922.12463 PMid:30079461

48. Sun Y, Xie G, Patel A, Li S, Zhao W, Yang X, et al. Prescription of statins at discharge and 1-year risk of major clinical outcomes among acute coronary syndromes patients with extremely low LDL-cholesterol in clinical pathways for acute coronary syndromes studies. Clin Cardiol. 2018;41(9):1192-200. https:// doi.org/10.1002/clc. 23040

PMid:30084224

49. Gencer B, Auer R, Nanchen D, Räber L, Klingenberg R,
Carballo D, et al. Expected impact of applying new 2013 AHA ACC cholesterol guidelines criteria on the recommended lipid target achievement after acute coronary syndromes. Atherosclerosis. 2015;239(1):118-24. https://doi.org/10.1016/j. atherosclerosis.2014.12.049

PMid:25585031

50. Bourdès V, Ferrières $\mathrm{J}$, Amar $\mathrm{J}$, Amelineau $\mathrm{E}$, Bonnevay $\mathrm{S}$, Berlion $\mathrm{M}$, et al. Prediction of persistence of combined evidence-based cardiovascular medications in patients with acute coronary syndrome after hospital discharge using neural networks. Med Biol Eng Comput. 2011;49(8):947-55. https://doi. org/10.1007/s11517-011-0785-4

PMid:21598000

51. Wang R, Neuenschwander FC, Lima Filho A, Moreira CM, Santos ES, Reis HJ, et al. Use of evidence-based interventions in acute coronary syndrome - Subanalysis of the ACCEPT registry. Arq Bras Cardiol. 2014;102(4):319-26. https://doi. org/10.5935/abc.20140033

PMid:24652052

52. Desta DM, Nedi T, Hailu A, Atey TM, Tsadik AG, Asgedom SW, et al. Treatment outcome of acute coronary syndrome patients admitted to Ayder Comprehensive Specialized Hospital, Mekelle, Ethiopia; A retrospective cross-sectional study. PLoS One. 2020;15(2):e0228953. https://doi.org/10.1371/journal. pone. 0228953

PMid:32053702

53. Andrikopoulos G, Tzeis S, Nikas N, Richter D, Pipilis A, Gotsis A, et al. Short-term outcome and attainment of secondary prevention goals in patients with acute coronary syndrome-results from the countrywide TARGET study. Int J Cardiol. 2013;168(2):922-7. https://doi.org/10.1016/j.ijcard.2012.10.049 PMid:23186596

54. Pereira M, Araújo C, Dias P, Lunet N, Subirana I, Marrugat J, et al. Age and sex inequalities in the prescription of evidence-based pharmacological therapy following an acute coronary syndrome in Portugal: The EURHOBOP study. Eur J Prev Cardiol. 2014;21(11):1401-8. https://doi. org/10.1177/2047487313494580

PMid:23787795

55. Huffman MD, Prabhakaran D, Abraham AK, Krishnan MN, Nambiar AC, Mohanan PP. Optimal in-hospital and discharge medical therapy in acute coronary syndromes in Kerala: Results from the Kerala acute coronary syndrome registry. Circ Cardiovasc Qual Outcomes. 2013;6(4):436-43. https://doi. org/10.1161/circoutcomes.113.000189 PMid:23800985

56. Ozawa S, Shankar R, Leopold C, Orubu S. Access to medicines through health systems in low- and middle-income countries. Health Policy Plan. 2019;34:iii1-3. https://doi.org/10.1093/ heapol/czz119

57. De Vera MA, Bhole V, Burns LC, Lacaille D. Impact of statin adherence on cardiovascular disease and mortality outcomes: A systematic review. Br J Clin Pharmacol. 2014;78(4):684-98. https://doi.org/10.1111/bcp.12339 PMid:25364801

58. Simpson RJ Jr., Mendys P. The effects of adherence and persistence on clinical outcomes in patients treated with statins: A systematic review. J Clin Lipidol. 2010;4(6):462-71. https://doi. org/10.1016/j.jacl.2010.08.026

PMid:21122692

59. Huber CA, Meyer MR, Steffel J, Blozik E, Reich O, Rosemann T. Post-myocardial infarction (MI) care: Medication adherence for secondary prevention after $\mathrm{MI}$ in a large real-world population. Clin Ther. 2019;41(1):107-17. https://doi.org/10.1016/j. clinthera.2018.11.012

PMid:30591287 
60. Lemstra M, Blackburn D, Crawley A, Fung R. Proportion and risk indicators of nonadherence to statin therapy: A meta-analysis. Can J Cardiol. 2012;28(5):574-80. https://doi.org/10.1016/j. cjca.2012.05.007

PMid:22884278

61. Mathews R, Wang TY, Honeycutt E, Henry TD, Zettler M, Chang $\mathrm{M}$, et al. Persistence with secondary prevention medications after acute myocardial infarction: Insights from the TRANSLATE-ACS study. Am Heart J. 2015;170(1):62-9. https:// doi.org/10.1016/j.ahj.2015.03.019

PMid:26093865

62. Gencer B, Rodondi N, Auer R, Räber L, Klingenberg R, Nanchen $\mathrm{D}$, et al. Reasons for discontinuation of recommended therapies according to the patients after acute coronary syndromes. Eur J Intern Med. 2015;26(1):56-62. https://doi. org/10.1016/j.ejim.2014.12.014

PMid:25582072

63. Akhter N, Milford-Beland S, Roe MT, Piana RN, Kao J, Shroff A. Gender differences among patients with acute coronary syndromes undergoing percutaneous coronary intervention in the American College of Cardiology-National Cardiovascular Data Registry (ACC-NCDR). Am Heart J. 2009;157(1):141-8. https://doi.org/10.1016/j.ahj.2008.08.012

PMid: 19081410

64. Tamargo J, Rosano G, Walther T, Duarte J, Niessner A, Kaski JC, et al. Gender differences in the effects of cardiovascular drugs. Eur Heart J Cardiovasc Pharmacother. 2017;3(3):163-82. https://doi.org/10.1093/ehjcvp/pvw042 PMid:28329228

65. Yang HY, Huang JH, Hsu CY, Chen YJ. Gender differences and the trend in the acute myocardial infarction: A 10-year nationwide population-based analysis. ScientificWorldJournal. 2012;2012:184075. PMid:22997490

66. Amsterdam EA, Wenger NK, Brindis RG, Casey DE, Ganiats TG, Holmes DR, et al. 2014 AHA/ACC guideline for the management of patients with non-ST-elevation acute coronary syndromes: Executive summary: A report of the American
College of Cardiology/American Heart Association Task Force on Practice Guidelines. J Am Coll Cardiol. 2014;130(25):235494. https://doi.org/10.1161/cir.0000000000000133 PMid:25249586

67. Grundy SM, Stone NJ, Bailey AL, Beam C, Birtcher KK, Blumenthal RS, et al. 2018 AHA/ACC/AACVPR/AAPA/ABC/ ACPM/ADA/AGS/APhA/ASPC/NLA/PCNA guideline on the management of blood cholesterol: A report of the American College of Cardiology/American Heart Association Task Force on Clinical Practice Guidelines. J Am Coll Cardiol. 2019;73(24):e285350. https://doi.org/10.1161/cir.0000000000000700 PMid:30423393

68. Deedwania PC. Statins in chronic kidney disease: Cardiovascular risk and kidney function. Postgrad Med. 2014;126(1):29-36. https://doi.org/10.3810/pgm.2014.01.2722 PMid:24393749

69. Ward NC, Watts GF, Eckel RH. Statin toxicity. Circ Res. 2019;124(2):328-50

PMid: 30653440

70. Swallow DM, Lawton MA, Grosset KA, Malek N, Klein J, Baig F, et al. Statins are underused in recent-onset Parkinson's disease with increased vascular risk: Findings from the UK Tracking Parkinson's and Oxford Parkinson's Disease Centre (OPDC) discovery cohorts. J Neurol Neurosurg Psychiatry. 2016;87(11):1183-90. https://doi.org/10.1136/jnnp-2016-313642 PMid:27671901

71. Grey C, Jackson R, Wells S, Thornley S, Marshall R, Crengle $S$, et al. Maintenance of statin use over 3 years following acute coronary syndromes: A national data linkage study (ANZACS-QI-2). Heart. 2014;100(10):770-4. https://doi. org/10.1136/heartjnl-2013-304960

PMid:24436219

72. Zeymer U, James S, Berkenboom G, Mohacsi A, Iniguez A, Coufal $Z$, et al. Differences in the use of guideline-recommended therapies among 14 European countries in patients with acute coronary syndromes undergoing $\mathrm{PCl}$. Eur J Prev Cardiol. 2013;20(2):218-28. https://doi.org/10.1177/2047487312437060 PMid:22345684 